Please share your stories about how Open Access to this article benefits you.

\title{
A non-overlapping domain decomposition method for incompressible Stokes equations with continuous pressures
}

\author{
by Jing Li and Xuemin Tu
}

2013

This is the published version of the article, made available with the permission of the publisher. The original published version can be found at the link below.

Jing Li, Xuemin Tu. (2013). A non-overlapping domain decomposition method for incompressible Stokes equations with continuous pressures. SIAM Journal on Numerical Analysis 51:1235-1253.

Published version: http://www.dx.doi.org/10.1137/120861503

Terms of Use: http://www2.ku.edu/ scholar/docs/license.shtml 


\title{
A NONOVERLAPPING DOMAIN DECOMPOSITION METHOD FOR INCOMPRESSIBLE STOKES EQUATIONS WITH CONTINUOUS PRESSURES*
}

\author{
JING $\mathrm{LI}^{\dagger}$ AND XUEMIN TU
}

\begin{abstract}
A nonoverlapping domain decomposition algorithm is proposed to solve the linear system arising from mixed finite element approximation of incompressible Stokes equations. A continuous finite element space for the pressure is used. In the proposed algorithm, Lagrange multipliers are used to enforce continuity of the velocity component across the subdomain boundary. The continuity of the pressure component is enforced in the primal form, i.e., neighboring subdomains share the same pressure degrees of freedom on the subdomain interface and no Lagrange multipliers are needed. After eliminating all velocity variables and the independent subdomain interior parts of the pressures, a symmetric positive semidefinite linear system for the subdomain boundary pressures and the Lagrange multipliers is formed and solved by a preconditioned conjugate gradient method. A lumped preconditioner is studied and the condition number bound of the preconditioned operator is proved to be independent of the number of subdomains for fixed subdomain problem size. Numerical experiments demonstrate the convergence rate of the proposed algorithm.
\end{abstract}

Key words. domain decomposition, incompressible Stokes, FETI-DP, BDDC

AMS subject classifications. 65F10,65N30, 65N55

DOI. $10.1137 / 120861503$

1. Introduction. Domain decomposition methods have been well studied for solving incompressible Stokes equations and similar saddle-point problems; see, e.g., $[16,24,20,10,3,22,11,28,29,25]$. In many of these works, special care is taken to deal with the divergence-free constraints across subdomain boundaries, which often lead to large coarse level problems. The large coarse level problem will be a bottleneck in large-scale parallel computations, and additional efforts in the algorithm are needed to reduce its impact; cf. [31, 32, 30, 17, 4, 15, 33]. Some recent progress has been made by Dohrmann and Widlund [5,6] for almost incompressible elasticity, where the coarse level space is built from discrete subdomain saddle-point harmonic extensions of certain subdomain interface cutoff functions and its dimension is much smaller than those in previous studies. Kim and Lee $[13,14]$ and Kim, Lee, and Park [12] studied the FETI-DP and BDDC algorithms for incompressible Stokes equations where a lumped preconditioner is used and reduction in the dimension of the coarse level space is also achieved.

In most of the above mentioned applications and analysis of domain decomposition methods for incompressible Stokes equations, the mixed finite element space contains discontinuous pressures. Application of discontinuous pressures in domain decomposition methods is natural. The decomposition of the pressure components among independent subdomains can be handled conveniently and no continuity of pressures across the subdomain boundary needs to be enforced. However, a big class

*Received by the editors January 5, 2012; accepted for publication (in revised form) February 4, 2013; published electronically April 23, 2013.

http://www.siam.org/journals/sinum/51-2/86150.html

${ }^{\dagger}$ Department of Mathematical Sciences, Kent State University, Kent, OH 44242 (li@math.kent. edu, http://www.math.kent.edu/ li/).

${ }_{\ddagger}^{\ddagger}$ Department of Mathematics, University of Kansas, Lawrence, KS 66045-7594 (xtu@math.ku.edu, http://www.math.ku.edu/ xtu/). This author's work was supported in part by National Science Foundation contract DMS-1115759. 
of mixed finite elements used for solving incompressible Stokes and Navier-Stokes equations have continuous pressures, e.g., the well-known Taylor-Hood finite elements type [27]. There have been some approaches using continuous pressures in domain decomposition methods for solving incompressible Stokes equations, e.g., by Klawonn and Pavarino [16], by Goldfeld [9], by Šístek et al. [26], and by Benhassine and Bendali [1]. In their work, an indefinite system of linear equations need to be solved, either by a generalized minimal residual method or simply by a conjugate gradient (CG) method. To the best of our knowledge, no scalable convergence rate has been proved analytically for any of those approaches using continuous pressures.

In this paper, we propose a nonoverlapping domain decomposition algorithm for solving incompressible Stokes equations with continuous pressure finite element space. The scalability of its convergence rate is proved. In this algorithm, the subdomain boundary velocities are dealt with in the same way as in the FETI-DP method: a few from each subdomain are selected as the coarse level primal variables, which are shared by neighboring subdomains; the others are local to subdomains and Lagrange multipliers are used to enforce their continuity. The subdomain boundary pressure degrees of freedom are all in primal form. They are shared by neighboring subdomains and no Lagrange multipliers are needed for their continuity. After eliminating all velocity variables and the independent subdomain interior parts of the pressures, the system for the subdomain boundary pressures and the Lagrange multipliers is shown to be symmetric positive semidefinite, which is solved by using a preconditioned $\mathrm{CG}$ method. Condition number bounds as strong as for the scalar elliptic case are established.

Only the application of a lumped preconditioner in the proposed algorithm is studied in this paper. The lumped preconditioner was first used in the FETI algorithm [7] for solving positive definite elliptic problems. Compared with the Dirichlet preconditioner, also used for the FETI algorithm [8], the lumped preconditioner is less effective in the reduction of the iteration count, but it is also less expensive in the computational cost for each iteration step. The main operation in the lumped preconditioner is subdomain matrix and vector products, while the implementation of the Dirichlet preconditioner requires solving subdomain systems of equations. For using the lumped preconditioner in the proposed algorithm to solve incompressible Stokes problems, the coarse level space can be chosen the same as that for solving scalar elliptic problems corresponding to each velocity component for both two- and three-dimensional problems. The additional subdomain edge and/or face components in the coarse space corresponding to the divergence-free constraints across subdomain boundaries, as discussed in [22], are not needed here, but they are needed when the Dirichlet preconditioner is used in the proposed algorithm, which is addressed in [34] by the authors. To stay focused on the purpose of this paper, the discussion of the proposed algorithm and its analysis are based on two-dimensional problems; the same approach can be extended to the three-dimensional case without substantial obstacles, which will be briefly discussed in several remarks in this paper. It is also worth pointing out that the domain decomposition algorithm and its analysis presented in this paper apply equally well, with only minor modifications, to the case where discontinuous pressures are used in the mixed finite element space.

The remainder of this paper is organized as follows. The finite element discretization of the incompressible Stokes equation is introduced in section 2. A domain decomposition approach is described in section 3. The system for the subdomain boundary pressures and the Lagrange multipliers is derived in section 4 . Section 5 provides some techniques used in the condition number bound estimate. In section 6 , 


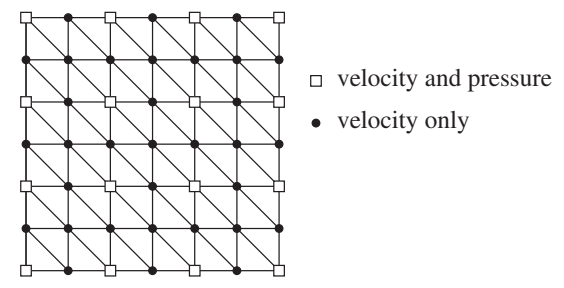

FIG. 2.1. A modified Taylor-Hood mixed finite element.

a lumped preconditioner is proposed and a scalable condition number bound of the preconditioned operator is established. At the end, in section 7, numerical results for solving a two-dimensional incompressible Stokes problem are shown to demonstrate the convergence rate of the proposed algorithm.

2. Finite element discretization. We consider solving the following incompressible Stokes problem on a bounded, two-dimensional polygonal domain $\Omega$ with a Dirichlet boundary condition:

$$
\left\{\begin{aligned}
-\Delta \mathbf{u}^{*}+\nabla p^{*} & =\mathbf{f} & & \text { in } \Omega, \\
-\nabla \cdot \mathbf{u}^{*} & =0 & & \text { in } \Omega, \\
\mathbf{u}^{*} & =\mathbf{u}_{\partial \Omega}^{*} & & \text { on } \partial \Omega,
\end{aligned}\right.
$$

where the boundary data $\mathbf{u}_{\partial \Omega}^{*}$ satisfies the compatibility condition $\int_{\partial \Omega} \mathbf{u}_{\partial \Omega}^{*} \cdot \mathbf{n}=0$. For simplicity, we assume that $\mathbf{u}_{\partial \Omega}^{*}=\mathbf{0}$ without losing any generality.

The weak solution of (2.1) is given by the following: find $\mathbf{u}^{*} \in\left(H_{0}^{1}(\Omega)\right)^{2}=\{\mathbf{v} \in$ $\left(H^{1}(\Omega)\right)^{2} \mid \mathbf{v}=\mathbf{0}$ on $\left.\partial \Omega\right\}$ and $p^{*} \in L^{2}(\Omega)$ such that

$$
\left\{\begin{aligned}
a\left(\mathbf{u}^{*}, \mathbf{v}\right)+b\left(\mathbf{v}, p^{*}\right) & =(\mathbf{f}, \mathbf{v}) & & \forall \mathbf{v} \in\left(H_{0}^{1}(\Omega)\right)^{2}, \\
b\left(\mathbf{u}^{*}, q\right) & =0 & & \forall q \in L^{2}(\Omega),
\end{aligned}\right.
$$

where $a\left(\mathbf{u}^{*}, \mathbf{v}\right)=\int_{\Omega} \nabla \mathbf{u}^{*} \cdot \nabla \mathbf{v}, b\left(\mathbf{u}^{*}, q\right)=-\int_{\Omega}\left(\nabla \cdot \mathbf{u}^{*}\right) q,(\mathbf{f}, \mathbf{v})=\int_{\Omega} \mathbf{f} \cdot \mathbf{v}$. We note that the solution of $(2.2)$ is not unique with the pressure $p^{*}$ different up to an additive constant.

A modified Taylor-Hood mixed finite element is used in this paper to solve (2.2). The domain $\Omega$ is triangulated into shape-regular elements of characteristic size $h$. The pressure finite element space, $Q \subset L^{2}(\Omega)$, is taken as the space of continuous piecewise linear functions on the triangulation. The velocity finite element space, $\mathbf{W} \subset\left(H_{0}^{1}(\Omega)\right)^{2}$, is formed by the continuous piecewise linear functions on the finer triangulation obtained by dividing each triangle into four subtriangles and connecting the middle points of its edges. A demonstration of this mixed finite element on a triangulation of a square domain is shown in Figure 2.1.

The finite element solution $(\mathbf{u}, p) \in \mathbf{W} \bigoplus Q$ of (2.2) satisfies

$$
\left[\begin{array}{cc}
A & B^{T} \\
B & 0
\end{array}\right]\left[\begin{array}{l}
\mathbf{u} \\
p
\end{array}\right]=\left[\begin{array}{l}
\mathbf{f} \\
0
\end{array}\right]
$$

where $A, B$, and $\mathbf{f}$ represent respectively the restrictions of $a(\cdot, \cdot), b(\cdot, \cdot)$, and $(\mathbf{f}, \cdot)$ to the finite-dimensional spaces $\mathbf{W}$ and $Q$. We use the same notation in this paper to represent both a finite element function and the vector of its nodal values. 
The coefficient matrix in (2.3) is rank deficient. $A$ is symmetric positive definite. The kernel of $B^{T}$, denoted by $\operatorname{Ker}\left(B^{T}\right)$, is the space of all constant pressures in $Q$. The range of $B$, denoted by $\operatorname{Im}(B)$, is orthogonal to $\operatorname{Ker}\left(B^{T}\right)$ and is the subspace of $Q$ consisting of all vectors with zero average. The solution of (2.3) always exists and is uniquely determined when the pressure is considered in the quotient space $Q / \operatorname{Ker}\left(B^{T}\right)$. In this paper, when $q \in Q / \operatorname{Ker}\left(B^{T}\right), q$ always has zero average. For a more general right-hand-side vector $(\mathbf{f}, g)$ given in (2.3), the existence of its solution requires that $g \in \operatorname{Im}(B)$, i.e., $g$ has zero average.

The modified Taylor-Hood mixed finite element space $\mathbf{W} \times Q$, as shown in Figure 2.1, is inf-sup stable in the sense that there exists a positive constant $\beta$, independent of $h$, such that

$$
\sup _{\mathbf{w} \in \mathbf{W}} \frac{b(\mathbf{w}, q)}{|\mathbf{w}|_{H^{1}}} \geq \beta\|q\|_{L^{2}} \quad \forall q \in Q / \operatorname{Ker}\left(B^{T}\right)
$$

(cf. [2, Chapter III, section 7]), or equivalently in matrix/vector form,

$$
\sup _{\mathbf{w} \in \mathbf{W}} \frac{\langle q, B \mathbf{w}\rangle^{2}}{\langle\mathbf{w}, A \mathbf{w}\rangle} \geq \beta^{2}\langle q, Z q\rangle \quad \forall q \in Q / \operatorname{Ker}\left(B^{T}\right) .
$$

Here, as always used in this paper, $\langle\cdot, \cdot\rangle$ represents the inner product of two vectors. The matrix $Z$ represents the mass matrix defined on the pressure finite element space $Q$, i.e., for any $q \in Q,\|q\|_{L^{2}}^{2}=\langle q, Z q\rangle$. It is easy to see (cf. [35, Lemma B.31]) that $Z$ is spectrally equivalent to $h^{2} I$ for two-dimensional problems, where $I$ represents the identity matrix of the same dimension, i.e., there exist positive constants $c$ and $C$, such that

$$
c h^{2} I \leq Z \leq C h^{2} I
$$

Here, as in other places of this paper, $c$ and $C$ represent generic positive constants which are independent of the mesh size $h$ and the subdomain diameter $H$ (discussed in the following section).

3. A nonoverlapping domain decomposition approach. The domain $\Omega$ is decomposed into $N$ nonoverlapping polygonal subdomains $\Omega_{i}, i=1,2, \ldots, N$. Each subdomain is the union of a bounded number of elements with the diameter of the subdomain in the order of $H$. The nodes on the interface of neighboring subdomains match across the subdomain boundaries $\Gamma=\left(\cup \partial \Omega_{i}\right) \backslash \partial \Omega$. $\Gamma$ is composed of subdomain edges, which are regarded as open subsets of $\Gamma$, and of the subdomain vertices, which are end points of edges.

The velocity and pressure finite element spaces $\mathbf{W}$ and $Q$ are decomposed into

$$
\mathbf{W}=\mathbf{W}_{I} \bigoplus \mathbf{W}_{\Gamma}, \quad Q=Q_{I} \bigoplus Q_{\Gamma},
$$

where $\mathbf{W}_{I}$ and $Q_{I}$ are direct sums of independent subdomain interior velocity spaces $\mathbf{W}_{I}^{(i)}$ and interior pressure spaces $Q_{I}^{(i)}$, respectively, i.e.,

$$
\mathbf{W}_{I}=\bigoplus_{i=1}^{N} \mathbf{W}_{I}^{(i)}, \quad Q_{I}=\bigoplus_{i=1}^{N} Q_{I}^{(i)} .
$$

$\mathbf{W}_{\Gamma}$ and $Q_{\Gamma}$ are subdomain boundary velocity and pressure spaces, respectively. All functions in $\mathbf{W}_{\Gamma}$ and $Q_{\Gamma}$ are continuous across the subdomain boundaries $\Gamma$; their degrees of freedom are shared by neighboring subdomains.

To formulate our domain decomposition algorithm, we introduce a partially subassembled subdomain boundary velocity space $\widetilde{\mathbf{W}}_{\Gamma}$, 


$$
\widetilde{\mathbf{W}}_{\Gamma}=\mathbf{w}_{\Pi} \bigoplus \mathbf{w}_{\Delta}=\mathbf{w}_{\Pi} \bigoplus\left(\bigoplus_{i=1}^{N} \mathbf{w}_{\Delta}^{(i)}\right) .
$$

$\mathbf{W}_{\Pi}$ is the continuous, coarse level, primal velocity space which is typically spanned by subdomain vertex nodal basis functions, and/or by interface edge-average basis functions with constant values, or with values of positive weights on these edges. The primal, coarse level velocity degrees of freedom are shared by neighboring subdomains. For the lumped preconditioner used in this paper, the coarse level velocity space can be chosen the same as for solving scalar elliptic problems corresponding to each velocity component. For example, $\mathbf{W}_{\Pi}$ can be as simple as spanned by subdomain vertex nodal velocity basis functions. The additional coarse level components across the subdomain edges, as discussed in [22], are no longer necessary here, even though including them in the coarse level space can improve the convergence rate of the proposed algorithm in terms of better stability of a certain subdomain jump operator; see Lemma 5.5.

The complementary space $\mathbf{W}_{\Delta}$ is the direct sum of independent subdomain dual interface velocity spaces $\mathbf{W}_{\Delta}^{(i)}$, which correspond to the remaining subdomain boundary velocity degrees of freedom and are spanned by basis functions which vanish at the primal degrees of freedom. Thus, an element in the space $\widetilde{\mathbf{W}}_{\Gamma}$ typically has a continuous primal velocity component and a discontinuous dual velocity component.

The functions $\mathbf{w}_{\Delta}$ in $\mathbf{W}_{\Delta}$ are in general not continuous across $\Gamma$. To enforce their continuity, we define a boolean matrix $B_{\Delta}$ constructed from $\{0,1,-1\}$. On each row of $B_{\Delta}$, there are only two nonzero entries, 1 and -1 , corresponding to the same velocity degree of freedom on each subdomain boundary node but attributed to two neighboring subdomains, such that for any $\mathbf{w}_{\Delta}$ in $\mathbf{W}_{\Delta}$, each row of $B_{\Delta} \mathbf{w}_{\Delta}=0$ implies that these two degrees of freedom from the two neighboring subdomains be the same. When nonredundant continuity constraints are enforced, $B_{\Delta}$ has full row rank. We denote the range of $B_{\Delta}$ applied on $\mathbf{W}_{\Delta}$ by $\Lambda$, the vector space of the Lagrange multipliers.

In order to define a certain subdomain boundary scaling operator, we introduce a positive scaling factor $\delta^{\dagger}(x)$ for each node $x$ on the subdomain boundary $\Gamma$. Let $\mathcal{N}_{x}$ be the number of subdomains sharing $x$, and we simply take $\delta^{\dagger}(x)=1 / \mathcal{N}_{x}$. In applications, these scaling factors will depend on the heat conduction coefficient for scalar elliptic problems and on the first of the Lamé parameters for the equation of linear elasticity, respectively; see [19, 18]. Given such scaling factors at the subdomain boundary nodes, we can define a scaled operator $B_{\Delta, D}$. We note that each row of $B_{\Delta}$ has only two nonzero entries, 1 and -1 , corresponding to the same subdomain boundary node $x$. Multiplying each entry by the scaling factor $\delta^{\dagger}(x)$ gives us $B_{\Delta, D}$.

Solving the original fully assembled linear system (2.3) is then equivalent to the following: find $\left(\mathbf{u}_{I}, p_{I}, \mathbf{u}_{\Delta}, \mathbf{u}_{\Pi}, p_{\Gamma}, \lambda\right) \in \mathbf{W}_{I} \oplus Q_{I} \oplus \mathbf{W}_{\Delta} \oplus \mathbf{W}_{\Pi} \oplus Q_{\Gamma} \oplus \Lambda$ such that

$$
\left[\begin{array}{cccccc}
A_{I I} & B_{I I}^{T} & A_{I \Delta} & A_{I \Pi} & B_{\Gamma I}^{T} & 0 \\
B_{I I} & 0 & B_{I \Delta} & B_{I \Pi} & 0 & 0 \\
A_{\Delta I} & B_{I \Delta}^{T} & A_{\Delta \Delta} & A_{\Delta \Pi} & B_{\Gamma \Delta}^{T} & B_{\Delta}^{T} \\
A_{\Pi I} & B_{I \Pi}^{T} & A_{\Pi \Delta} & A_{\Pi \Pi} & B_{\Gamma \Pi}^{T} & 0 \\
B_{\Gamma I} & 0 & B_{\Gamma \Delta} & B_{\Gamma \Pi} & 0 & 0 \\
0 & 0 & B_{\Delta} & 0 & 0 & 0
\end{array}\right]\left[\begin{array}{c}
\mathbf{u}_{I} \\
p_{I} \\
\mathbf{u}_{\Delta} \\
\mathbf{u}_{\Pi} \\
p_{\Gamma} \\
\lambda
\end{array}\right]=\left[\begin{array}{c}
\mathbf{f}_{I} \\
0 \\
\mathbf{f}_{\Delta} \\
\mathbf{f}_{\Pi} \\
0 \\
0
\end{array}\right],
$$


where the subblocks in the coefficient matrix represent the restrictions of $A$ and $B$ in (2.3) to appropriate subspaces. The leading three-by-three block can be made block diagonal with each diagonal block representing one independent subdomain problem.

Corresponding to the one-dimensional null space of (2.3), we consider a vector of the form $\left(\mathbf{u}_{I}, p_{I}, \mathbf{u}_{\Delta}, \mathbf{u}_{\Pi}, p_{\Gamma}, \lambda\right)=\left(\mathbf{0}, 1_{p_{I}}, \mathbf{0}, \mathbf{0}, 1_{p_{\Gamma}}, \lambda\right)$, where $1_{p_{I}} \in Q_{I}$ and $1_{p_{\Gamma}} \in Q_{\Gamma}$ represent vectors with value 1 on each entry. Substituting it into (3.1) gives zero blocks on the right-hand side, except that at the third block the right-hand side equals

$$
\left[\begin{array}{ll}
B_{I \Delta}^{T} & B_{\Gamma \Delta}^{T}
\end{array}\right]\left[\begin{array}{c}
1_{p_{I}} \\
1_{p_{\Gamma}}
\end{array}\right]+B_{\Delta}^{T} \lambda
$$

The first term represents the line integral of the normal component of the velocity finite element basis functions across the subdomain boundary on neighboring subdomains. Corresponding to the same subdomain boundary velocity degree of freedom, their values on the two neighboring subdomains are negative to each other, and the values are unchanged under the application of $B_{\Delta}^{T} B_{\Delta, D}$, which essentially computes the jump of a function across the subdomain boundary. Therefore

$$
\left[\begin{array}{ll}
B_{I \Delta}^{T} & B_{\Gamma \Delta}^{T}
\end{array}\right]\left[\begin{array}{c}
1_{p_{I}} \\
1_{p_{\Gamma}}
\end{array}\right]=B_{\Delta}^{T} B_{\Delta, D}\left\{\left[B_{I \Delta}^{T} \quad B_{\Gamma \Delta}^{T}\right]\left[\begin{array}{l}
1_{p_{I}} \\
1_{p_{\Gamma}}
\end{array}\right]\right\}
$$

from which we know that, taking

$$
\lambda=-B_{\Delta, D}\left[\begin{array}{ll}
B_{I \Delta}^{T} & B_{\Gamma \Delta}^{T}
\end{array}\right]\left[\begin{array}{c}
1_{p_{I}} \\
1_{p_{\Gamma}}
\end{array}\right],
$$

the right-hand-side block (3.2) equals $\mathbf{0}$. Therefore, a basis of the one-dimensional null space of (3.1) is

$$
\left(\begin{array}{llllll}
0, & 1_{p_{I}}, & 0, & 0, & 1_{p_{\Gamma}}, & -B_{\Delta, D}
\end{array}\left[\begin{array}{ll}
B_{I \Delta}^{T} & B_{\Gamma \Delta}^{T}
\end{array}\right]\left[\begin{array}{c}
1_{p_{I}} \\
1_{p_{\Gamma}}
\end{array}\right]\right) .
$$

4. A reduced symmetric positive semidefinite system. The system (3.1) can be reduced to a Schur complement problem for the variables $\left(p_{\Gamma}, \lambda\right)$. Since the leading four-by-four block of the coefficient matrix in (3.1) is invertible, the variables $\left(\mathbf{u}_{I}, p_{I}, \mathbf{u}_{\Delta}, \mathbf{u}_{\Pi}\right)$ can be eliminated and we obtain

$$
G\left[\begin{array}{c}
p_{\Gamma} \\
\lambda
\end{array}\right]=g,
$$

where

$$
G=\left[\begin{array}{cccc}
B_{\Gamma I} & 0 & B_{\Gamma \Delta} & B_{\Gamma \Pi} \\
0 & 0 & B_{\Delta} & 0
\end{array}\right]\left[\begin{array}{cccc}
A_{I I} & B_{I I}^{T} & A_{I \Delta} & A_{I \Pi} \\
B_{I I} & 0 & B_{I \Delta} & B_{I \Pi} \\
A_{\Delta I} & B_{I \Delta}^{T} & A_{\Delta \Delta} & A_{\Delta \Pi} \\
A_{\Pi I} & B_{I \Pi}^{T} & A_{\Pi \Delta} & A_{\Pi \Pi}
\end{array}\right]^{-1}\left[\begin{array}{cc}
B_{\Gamma I}^{T} & 0 \\
0 & 0 \\
B_{\Gamma \Delta}^{T} & B_{\Delta}^{T} \\
B_{\Gamma \Pi}^{T} & 0
\end{array}\right]
$$

and

$$
g=\left[\begin{array}{cccc}
B_{\Gamma I} & 0 & B_{\Gamma \Delta} & B_{\Gamma \Pi} \\
0 & 0 & B_{\Delta} & 0
\end{array}\right]\left[\begin{array}{cccc}
A_{I I} & B_{I I}^{T} & A_{I \Delta} & A_{I \Pi} \\
B_{I I} & 0 & B_{I \Delta} & B_{I \Pi} \\
A_{\Delta I} & B_{I \Delta}^{T} & A_{\Delta \Delta} & A_{\Delta \Pi} \\
A_{\Pi I} & B_{I \Pi}^{T} & A_{\Pi \Delta} & A_{\Pi \Pi}
\end{array}\right]^{-1}\left[\begin{array}{c}
\mathbf{f}_{I} \\
0 \\
\mathbf{f}_{\Delta} \\
\mathbf{f}_{\Pi}
\end{array}\right] .
$$


We denote

$$
\widetilde{A}=\left[\begin{array}{cccc}
A_{I I} & B_{I I}^{T} & A_{I \Delta} & A_{I \Pi} \\
B_{I I} & 0 & B_{I \Delta} & B_{I \Pi} \\
A_{\Delta I} & B_{I \Delta}^{T} & A_{\Delta \Delta} & A_{\Delta \Pi} \\
A_{\Pi I} & B_{I \Pi}^{T} & A_{\Pi \Delta} & A_{\Pi \Pi}
\end{array}\right] \quad \text { and } \quad B_{C}=\left[\begin{array}{cccc}
B_{\Gamma I} & 0 & B_{\Gamma \Delta} & B_{\Gamma \Pi} \\
0 & 0 & B_{\Delta} & 0
\end{array}\right] .
$$

We can see that $-G$ is the Schur complement of the coefficient matrix of (3.1) with respect to the last two row blocks, i.e.,

$$
\left[\begin{array}{cc}
I & 0 \\
-B_{C} \widetilde{A}^{-1} & I
\end{array}\right]\left[\begin{array}{cc}
\widetilde{A} & B_{C}^{T} \\
B_{C} & 0
\end{array}\right]\left[\begin{array}{cc}
I & -\widetilde{A}^{-1} B_{C}^{T} \\
0 & I
\end{array}\right]=\left[\begin{array}{cc}
\widetilde{A} & 0 \\
0 & -G
\end{array}\right] .
$$

From the Sylvester law of inertia, namely, the number of positive, negative, and zero eigenvalues of a symmetric matrix is invariant under a change of coordinates, we can see that the number of zero eigenvalues of $G$ is the same as the number of zero eigenvalues (with multiplicity counted) of the original coefficient matrix of (3.1), which is one, and all other eigenvalues of $G$ are positive. Therefore $G$ is symmetric positive semidefinite. The null space of $G$ is derived from the null space of the original coefficient matrix of (3.1), and its basis is given by (cf. (3.3))

$$
\left(1_{p_{\Gamma}},-B_{\Delta, D}\left[B_{I \Delta}^{T} \quad B_{\Gamma \Delta}^{T}\right]\left[\begin{array}{l}
1_{p_{I}} \\
1_{p_{\Gamma}}
\end{array}\right]\right) .
$$

We denote $X=Q_{\Gamma} \oplus \Lambda$. The range of $G$, denoted by $R_{G}$, is the subspace of $X$ orthogonal to the null space of $G$ and has the form

$$
R_{G}=\left\{\left[\begin{array}{c}
g_{p_{\Gamma}} \\
g_{\lambda}
\end{array}\right] \in X \mid g_{p_{\Gamma}}^{T} 1_{p_{\Gamma}}-g_{\lambda}^{T}\left(B_{\Delta, D}\left[B_{I \Delta}^{T} \quad B_{\Gamma \Delta}^{T}\right]\left[\begin{array}{c}
1_{p_{I}} \\
1_{p_{\Gamma}}
\end{array}\right]\right)=0\right\} .
$$

The restriction of $G$ to its range $R_{G}$ is positive definite. The fact that the solution of (3.1) always exists for any given $\left(\mathbf{f}_{I}, \mathbf{f}_{\Delta}, \mathbf{f}_{\Pi}\right)$ on the right-hand side implies that the solution of (4.1) exists for any $g$ defined by (4.3). Therefore $g \in R_{G}$. When the CG method is applied to solve (4.1) with zero initial guess, all the iterates are in the Krylov subspace generated by $G$ and $g$, which is also a subspace of $R_{G}$, and where the CG cannot break down. After obtaining $\left(p_{\Gamma}, \lambda\right)$ from solving (4.1), the other components $\left(\mathbf{u}_{I}, p_{I}, \mathbf{u}_{\Delta}, \mathbf{u}_{\Pi}\right)$ in (3.1) are obtained by back substitution.

In the rest of this section, we discuss the implementation of multiplying $G$ by a vector. The main operation is the product of $\widetilde{A}^{-1}$ with a vector; cf. (4.2) and (4.3). We denote

$$
A_{r r}=\left[\begin{array}{ccc}
A_{I I} & B_{I I}^{T} & A_{I \Delta} \\
B_{I I} & 0 & B_{I \Delta} \\
A_{\Delta I} & B_{I \Delta}^{T} & A_{\Delta \Delta}
\end{array}\right], \quad A_{\Pi r}=A_{r \Pi}^{T}=\left[\begin{array}{lll}
A_{\Pi I} & B_{I \Pi}^{T} & A_{\Pi \Delta}
\end{array}\right], \quad f_{r}=\left[\begin{array}{l}
\mathbf{f}_{I} \\
0 \\
\mathbf{f}_{\Delta}
\end{array}\right]
$$

and define the Schur complement

$$
S_{\Pi}=A_{\Pi \Pi}-A_{\Pi r} A_{r r}^{-1} A_{r \Pi},
$$

which is symmetric positive definite from the Sylvester law of inertia. $S_{\Pi}$ defines the coarse level problem in the algorithm. The product 


$$
\left[\begin{array}{cccc}
A_{I I} & B_{I I}^{T} & A_{I \Delta} & A_{I \Pi} \\
B_{I I} & 0 & B_{I \Delta} & B_{I \Pi} \\
A_{\Delta I} & B_{I \Delta}^{T} & A_{\Delta \Delta} & A_{\Delta \Pi} \\
A_{\Pi I} & B_{I \Pi}^{T} & A_{\Pi \Delta} & A_{\Pi \Pi}
\end{array}\right]^{-1}\left[\begin{array}{l}
\mathbf{f}_{I} \\
0 \\
\mathbf{f}_{\Delta} \\
\mathbf{f}_{\Pi}
\end{array}\right]
$$

can then be represented by

$$
\left[\begin{array}{c}
A_{r r}^{-1} f_{r} \\
\mathbf{0}
\end{array}\right]+\left[\begin{array}{c}
-A_{r r}^{-1} A_{r \Pi} \\
I_{\Pi}
\end{array}\right] S_{\Pi}^{-1}\left(\mathbf{f}_{\Pi}-A_{\Pi r} A_{r r}^{-1} f_{r}\right),
$$

which requires solving the coarse level problem once and independent subdomain Stokes problems with Neumann type boundary conditions twice.

5. Preliminary results. We first define certain norms for several vector/function spaces. We denote

$$
\widetilde{\mathbf{W}}=\mathbf{W}_{I} \bigoplus \widetilde{\mathbf{W}}_{\Gamma}
$$

For any $\mathbf{w}$ in $\widetilde{\mathbf{W}}$, we denote its restriction to subdomain $\Omega_{i}$ by $\mathbf{w}^{(i)}$. A subdomainwise $H^{1}$-seminorm can be defined for functions in $\widetilde{\mathbf{W}}$ by

$$
|\mathbf{w}|_{H^{1}}^{2}=\sum_{i=1}^{N}\left|\mathbf{w}^{(i)}\right|_{H^{1}\left(\Omega_{i}\right)}^{2} .
$$

We also define

$$
\widetilde{V}=\mathbf{w}_{I} \bigoplus Q_{I} \bigoplus \mathbf{w}_{\Delta} \bigoplus \mathbf{w}_{\Pi}
$$

and its subspace

$$
\widetilde{V}_{0}=\left\{v=\left(\mathbf{w}_{I}, p_{I}, \mathbf{w}_{\Delta}, \mathbf{w}_{\Pi}\right) \in \widetilde{V} \mid B_{I I} \mathbf{w}_{I}+B_{I \Delta} \mathbf{w}_{\Delta}+B_{I \Pi} \mathbf{w}_{\Pi}=0\right\} .
$$

For any $v=\left(\mathbf{w}_{I}, p_{I}, \mathbf{w}_{\Delta}, \mathbf{w}_{\Pi}\right) \in \widetilde{V}_{0}$, let $\mathbf{w}=\left(\mathbf{w}_{I}, \mathbf{w}_{\Delta}, \mathbf{w}_{\Pi}\right) \in \widetilde{\mathbf{W}}$. Then

$$
\begin{aligned}
\langle v, v\rangle_{\widetilde{A}} & =\left[\begin{array}{c}
\mathbf{w}_{I} \\
\mathbf{w}_{\Delta} \\
\mathbf{w}_{\Pi}
\end{array}\right]^{T}\left[\begin{array}{ccc}
A_{I I} & A_{I \Delta} & A_{I \Pi} \\
A_{\Delta I} & A_{\Delta \Delta} & A_{\Delta \Pi} \\
A_{\Pi I} & A_{\Pi \Delta} & A_{\Pi \Pi}
\end{array}\right]\left[\begin{array}{c}
\mathbf{w}_{I} \\
\mathbf{w}_{\Delta} \\
\mathbf{w}_{\Pi}
\end{array}\right] \\
& =\sum_{i=1}^{N}\left[\begin{array}{c}
\mathbf{w}_{I}^{(i)} \\
\mathbf{w}_{\Delta}^{(i)} \\
\mathbf{w}_{\Pi}^{(i)}
\end{array}\right]^{T}\left[\begin{array}{ccc}
A_{I I}^{(i)} & A_{I \Delta}^{(i)} & A_{I \Pi}^{(i)} \\
A_{\Delta I}^{(i)} & A_{\Delta \Delta}^{(i)} & A_{\Delta \Pi}^{(i)} \\
A_{\Pi I}^{(i)} & A_{\Pi \Delta}^{(i)} & A_{\Pi \Pi}^{(i)}
\end{array}\right]\left[\begin{array}{c}
\mathbf{w}_{I}^{(i)} \\
\mathbf{w}_{\Delta}^{(i)} \\
\mathbf{w}_{\Pi}^{(i)}
\end{array}\right]=\sum_{i=1}^{N}\left|\left[\begin{array}{c}
\mathbf{w}_{I}^{(i)} \\
\mathbf{w}_{\Delta}^{(i)} \\
\mathbf{w}_{\Pi}^{(i)}
\end{array}\right]\right|_{H^{1}\left(\Omega_{i}\right)}^{2} \\
& =|\mathbf{w}|_{H^{1}}^{2}
\end{aligned}
$$

i.e., $\langle\cdot, \cdot\rangle_{\widetilde{A}}$ defines an inner product on $\widetilde{V}_{0}$. In (5.3), the superscript ${ }^{(i)}$ is used to represent the restrictions of corresponding vectors and matrices to subdomain $\Omega_{i}$.

Since $\mathbf{W}$ is essentially the subspace of $\widetilde{\mathbf{W}}$ with continuous subdomain boundary velocities, the inf-sup condition (2.4) and (2.5) also holds for the mixed space $\widetilde{\mathbf{W}} \times Q$. 
Denote

$$
\widetilde{B}=\left[\begin{array}{lll}
B_{I I} & B_{I \Delta} & B_{I \Pi} \\
B_{\Gamma I} & B_{\Gamma \Delta} & B_{\Gamma \Pi}
\end{array}\right], \quad \widetilde{A}_{\mathbf{u}}=\left[\begin{array}{lll}
A_{I I} & A_{I \Delta} & A_{I \Pi} \\
A_{\Delta I} & A_{\Delta \Delta} & A_{\Delta \Pi} \\
A_{\Pi I} & A_{\Pi \Delta} & A_{\Pi \Pi}
\end{array}\right],
$$

as in (3.1); then

$$
\sup _{\mathbf{w} \in \widetilde{\mathbf{W}}} \frac{\langle q, \widetilde{B} \mathbf{w}\rangle^{2}}{\left\langle\mathbf{w}, \widetilde{A}_{\mathbf{u}} \mathbf{w}\right\rangle} \geq \beta^{2}\langle q, Z q\rangle \quad \forall q \in Q / \operatorname{Ker}\left(B^{T}\right),
$$

where $\beta$ is the same as in (2.4) and (2.5).

We also have the following lemma on the stability of the operator $\widetilde{B}$.

Lemma 5.1. For any $\mathbf{w} \in \widetilde{\mathbf{W}}$ and $q \in Q,\langle\widetilde{B} \mathbf{w}, q\rangle \leq|\mathbf{w}|_{H^{1}}\|q\|_{L^{2}}$.

Proof.

$$
\begin{aligned}
\langle\widetilde{B} \mathbf{w}, q\rangle^{2} & =\left(\sum_{i=1}^{N} \int_{\Omega_{i}} \nabla \cdot \mathbf{w}^{(i)} q\right)^{2} \leq\left(\sum_{i=1}^{N} \sqrt{\int_{\Omega_{i}}\left|\nabla \mathbf{w}^{(i)}\right|^{2}} \sqrt{\int_{\Omega_{i}} q^{2}}\right)^{2} \\
& \leq\left(\sum_{i=1}^{N} \int_{\Omega_{i}}\left|\nabla \mathbf{w}^{(i)}\right|^{2}\right)\left(\sum_{i=1}^{N} \int_{\Omega_{i}} q^{2}\right)=|\mathbf{w}|_{H^{1}}^{2}\|q\|_{L^{2}}^{2} .
\end{aligned}
$$

The finite element space for subdomain boundary pressures, $Q_{\Gamma}$, is a subspace of $L^{2}(\Gamma)$. For each $p_{\Gamma} \in Q_{\Gamma}$, its finite element extension by zero to the interior of subdomains is denoted by $p_{\Gamma}^{E}$, which equals $p_{\Gamma}$ on all subdomain boundary nodes and equals zero on all subdomain interior nodes. We can see that $p_{\Gamma}^{E} \in Q \subset L^{2}(\Omega)$ and $\left\|p_{\Gamma}^{E}\right\|_{L^{2}(\Omega)}^{2}=\left\langle p_{\Gamma}^{E}, p_{\Gamma}^{E}\right\rangle_{Z}$ from the definition of $Z$ in section 2 .

From (4.2) and (4.4), we can see that

$$
G=B_{C} \widetilde{A}^{-1} B_{C}^{T}
$$

In particular, we denote the first row of $B_{C}$ by

$$
\widetilde{B}_{\Gamma}=\left[\begin{array}{llll}
B_{\Gamma I} & 0 & B_{\Gamma \Delta} & B_{\Gamma \Pi}
\end{array}\right] ;
$$

for the second row, we denote the restriction operator from $\widetilde{V}$ onto $\mathbf{W}_{\Delta}$ by $\widetilde{R}_{\Delta}$ such that for any $v=\left(\mathbf{w}_{I}, p_{I}, \mathbf{w}_{\Delta}, \mathbf{w}_{\Pi}\right) \in \widetilde{V}, \widetilde{R}_{\Delta} v=\mathbf{w}_{\Delta}$. Then $G$ can be represented by the two-by-two block structure

$$
G=\left[\begin{array}{cc}
G_{p_{\Gamma} p_{\Gamma}} & G_{p_{\Gamma} \lambda} \\
G_{\lambda p_{\Gamma}} & G_{\lambda \lambda}
\end{array}\right]
$$

where

$$
\begin{array}{cl}
G_{p_{\Gamma} p_{\Gamma}}=\widetilde{B}_{\Gamma} \widetilde{A}^{-1} \widetilde{B}_{\Gamma}^{T}, & G_{p_{\Gamma} \lambda}=\widetilde{B}_{\Gamma} \widetilde{A}^{-1} \widetilde{R}_{\Delta}^{T} B_{\Delta}^{T}, \\
G_{\lambda p_{\Gamma}}=B_{\Delta} \widetilde{R}_{\Delta} \widetilde{A}^{-1} \widetilde{B}_{\Gamma}^{T}, & G_{\lambda \lambda}=B_{\Delta} \widetilde{R}_{\Delta} \widetilde{A}^{-1} \widetilde{R}_{\Delta}^{T} B_{\Delta}^{T} .
\end{array}
$$

The pressure components of all vectors in $R_{G}$ with $g_{\lambda}=0$ (cf. (4.5)) form a subspace of $Q_{\Gamma}$ and we denote this subspace by $R_{G \mid Q_{\Gamma}}$. From the definition of $R_{G}$, 
we can see that for any vector $p_{\Gamma} \in R_{G \mid Q_{\Gamma}}, p_{\Gamma}^{T} 1_{p_{\Gamma}}=0$, and then its extension by zero to the interior of subdomains, $p_{\Gamma}^{E}$, also has zero average.

The following lemma follows essentially from [35, Lemma 9.1].

LeMmA 5.2. For all $p_{\Gamma} \in R_{G \mid Q_{\Gamma}}$,

$$
\beta^{2}\left\|p_{\Gamma}^{E}\right\|_{L^{2}(\Omega)}^{2} \leq\left\langle p_{\Gamma}, G_{p_{\Gamma} p_{\Gamma}} p_{\Gamma}\right\rangle \leq\left\|p_{\Gamma}^{E}\right\|_{L^{2}(\Omega)}^{2},
$$

where $p_{\Gamma}^{E}$ represents the extension by zero of $p_{\Gamma}$ to the interior of subdomains and $\beta$ is the same as in (2.4) and (2.5).

Proof. Note that even though $\widetilde{A}^{-1}$ is indefinite in $\widetilde{V}$, it is positive definite when restricted to a subspace of $\widetilde{V}$, where the pressure component equals zero, and the norm $\|\cdot\|_{\widetilde{A}^{-1}}$ is well defined.

To prove the left-side inequality, denote for any $\mathbf{v}=\left(\mathbf{v}_{I}, \mathbf{v}_{\Delta}, \mathbf{v}_{\Pi}\right) \in \widetilde{\mathbf{W}}, \mathbf{v}^{\dagger}=$ $\left(\mathbf{v}_{I}, 0, \mathbf{v}_{\Delta}, \mathbf{v}_{\Pi}\right) \in \widetilde{V}$. We have

$$
\begin{gathered}
\left\langle p_{\Gamma}, \widetilde{B}_{\Gamma} \widetilde{A}^{-1} \widetilde{B}_{\Gamma}^{T} p_{\Gamma}\right\rangle=\left\|\widetilde{B}_{\Gamma}^{T} p_{\Gamma}\right\|_{\widetilde{A}^{-1}}^{2}=\sup _{\mathbf{v} \in \widetilde{\mathbf{W}}} \frac{\left\langle\mathbf{v}^{\dagger}, \widetilde{B}_{\Gamma}^{T} p_{\Gamma}\right\rangle_{\widetilde{A}^{-1}}^{2}}{\left\|\mathbf{v}^{\dagger}\right\|_{\widetilde{A}^{-1}}^{2}}=\sup _{\mathbf{v} \in \widetilde{\mathbf{W}}} \frac{\left(p_{\Gamma}^{T} \widetilde{B}_{\Gamma} \widetilde{A}^{-1} \mathbf{v}^{\dagger}\right)^{2}}{\mathbf{v}^{\dagger} \widetilde{A}^{-1} \mathbf{v}^{\dagger}} \\
=\sup _{\mathbf{w} \in \widetilde{\mathbf{W}}} \frac{\left(p_{\Gamma}^{T} \widetilde{B}_{\Gamma} \mathbf{w}^{\dagger}\right)^{2}}{\mathbf{w}^{\dagger^{T}} \widetilde{A} \mathbf{w}^{\dagger}}=\sup _{\mathbf{w} \in \widetilde{\mathbf{W}}} \frac{\left(p_{\Gamma}^{E^{T}} \widetilde{B} \mathbf{w}\right)^{2}}{\mathbf{w}^{T} \widetilde{A}_{\mathbf{u}} \mathbf{w}} \geq \beta^{2}\left\langle p_{\Gamma}^{E}, p_{\Gamma}^{E}\right\rangle_{Z}=\beta^{2}\left\|p_{\Gamma}^{E}\right\|_{L^{2}(\Omega)}^{2},
\end{gathered}
$$

where we have used the inf-sup condition (5.5) for the inequality in the middle.

To prove the right-side inequality, for any given $p_{\Gamma} \in R_{G \mid Q_{\Gamma}}$, denote $\mathbf{v}^{\dagger}=$ $\left(\mathbf{v}_{I}, p_{I}, \mathbf{v}_{\Delta}, \mathbf{v}_{\Pi}\right)=\widetilde{A}^{-1} \widetilde{B}_{\Gamma}^{T} p_{\Gamma}$, and the shorter vector $\mathbf{v}=\left(\mathbf{v}_{I}, \mathbf{v}_{\Delta}, \mathbf{v}_{\Pi}\right)$. From the continuity of $\widetilde{B}$ in Lemma 5.1 and (5.3), we have

$$
\begin{aligned}
& \left\langle p_{\Gamma}, \widetilde{B}_{\Gamma} \widetilde{A}^{-1} \widetilde{B}_{\Gamma}^{T} p_{\Gamma}\right\rangle=\left\langle p_{\Gamma}, \widetilde{B}_{\Gamma} \mathbf{v}^{\dagger}\right\rangle=\left\langle p_{\Gamma}^{E}, \widetilde{B} \mathbf{v}\right\rangle \leq\left\|p_{\Gamma}^{E}\right\|_{L^{2}}|\mathbf{v}|_{H^{1}} \\
& \quad=\left\|p_{\Gamma}^{E}\right\|_{L^{2}} \sqrt{\left\langle\widetilde{A}^{-1} \widetilde{B}_{\Gamma}^{T} p_{\Gamma}, \widetilde{A}^{-1} \widetilde{B}_{\Gamma}^{T} p_{\Gamma}\right\rangle_{\widetilde{A}}}=\left\|p_{\Gamma}^{E}\right\|_{L^{2}}\left\langle p_{\Gamma}, \widetilde{B}_{\Gamma} \widetilde{A}^{-1} \widetilde{B}_{\Gamma}^{T} p_{\Gamma}\right\rangle^{1 / 2} .
\end{aligned}
$$

The following corollary of Lemma 5.2 is an immediate result from (2.6) and the facts that $\left\|p_{\Gamma}^{E}\right\|_{L^{2}(\Omega)}^{2}=\left\langle p_{\Gamma}^{E}, p_{\Gamma}^{E}\right\rangle_{Z},\left\langle p_{\Gamma}^{E}, p_{\Gamma}^{E}\right\rangle=\left\langle p_{\Gamma}, p_{\Gamma}\right\rangle$.

COROLlarY 5.3. There exist positive constants $c$ and $C$ such that

$$
c h^{2} \beta^{2} I_{p_{\Gamma}} \leq G_{p_{\Gamma} p_{\Gamma}} \leq C h^{2} I_{p_{\Gamma}},
$$

where $I_{p_{\Gamma}}$ is the identity matrix of the same dimension as $G_{p_{\Gamma} p_{\Gamma}}$ and $\beta$ is the same as in (2.4) and (2.5).

Remark 5.4. Lemma 5.2 and Corollary 5.3 are not used in our proof of the condition number bound in section 6 . However, it is intuitive to see from Corollary 5.3 that the first diagonal block $G_{p_{\Gamma} p_{\Gamma}}$ in matrix $G$ can be approximated spectrally equivalently by the identity matrix multiplied by $h^{2}$, which is what is being done in our block diagonal preconditioner discussed in section 6 .

We also need to define a certain jump operator across the subdomain boundaries $\Gamma$. Let $P_{D}: \widetilde{V} \rightarrow \widetilde{V}$ be defined by (cf. [21])

$$
P_{D}=\widetilde{R}_{\Delta}^{T} B_{\Delta, D}^{T} B_{\Delta} \widetilde{R}_{\Delta}
$$

Copyright (c) by SIAM. Unauthorized reproduction of this article is prohibited. 
We can see that application of $P_{D}$ to a vector essentially computes the difference (jump) of the dual velocity component across the subdomain boundaries and then distributes the jump to neighboring subdomains according to the scaling factor $\delta^{\dagger}(x)$. In fact, the dual velocity component is the only part of the vector involved in the application of $P_{D}$; all other components are kept zero and are added into the definition to make $P_{D}$ more convenient to use in the presentation of the algorithm. We also have, for any $v=\left(\mathbf{w}_{I}, p_{I}, \mathbf{w}_{\Delta}, \mathbf{w}_{\Pi}\right) \in \widetilde{V}$,

$$
\left\langle P_{D} v, P_{D} v\right\rangle_{\widetilde{A}}=\left\langle B_{\Delta, D}^{T} B_{\Delta} \mathbf{w}_{\Delta}, B_{\Delta, D}^{T} B_{\Delta} \mathbf{w}_{\Delta}\right\rangle_{A_{\Delta \Delta}} .
$$

The following lemma can be found essentially from [23, section 6.1]; see also (5.3).

LEMMA 5.5. There exists a function $\Phi(H / h)$ such that for all $v \in \widetilde{V}_{0}$,

$$
\left\langle P_{D} v, P_{D} v\right\rangle_{\widetilde{A}} \leq C \Phi(H / h)\langle v, v\rangle_{\widetilde{A}} .
$$

Here, for two-dimensional problems, $\Phi(H / h)=(H / h)(1+\log (H / h))$, when only subdomain corner velocities are chosen as coarse level primal variables; $\Phi(H / h)=$ $H / h$, when both subdomain corner and edge-average velocity degrees of freedom are chosen as primal variables.

Remark 5.6. For three-dimensional problems, just as for the positive definite elliptic problems discussed in [23, section 6.2], when both subdomain corner and edgeaverage velocities are chosen as primal variables, $\Phi(H / h)=(H / h)(1+\log (H / h))$.

The following lemma is also used and can be found at [10, Lemma 2.3].

Lemma 5.7. Consider the following saddle-point problem: find $(\mathbf{u}, p) \in \mathbf{W} \oplus Q$ such that

$$
\left[\begin{array}{cc}
A & B^{T} \\
B & 0
\end{array}\right]\left[\begin{array}{l}
\mathbf{u} \\
p
\end{array}\right]=\left[\begin{array}{l}
\mathbf{f} \\
g
\end{array}\right],
$$

where $A$ and $B$ are as in (2.3), $\mathbf{f} \in \mathbf{W}$, and $g \in \operatorname{Im}(B) \subset Q$. Let $\beta$ be the inf-sup constant specified in (2.5). Then

$$
\|\mathbf{u}\|_{A} \leq\|\mathbf{f}\|_{A^{-1}}+\frac{1}{\beta}\|g\|_{Z^{-1}}
$$

where $Z$ is the mass matrix defined in section 2.

6. A lumped preconditioner. We consider a block diagonal preconditioner for (4.1). From Corollary 5.3, the inverse of the first diagonal block $G_{p_{\Gamma} p_{\Gamma}}$ of $G$ can be effectively approximated by $1 / h^{2}$ times the identity matrix. The inverse of the second diagonal block $B_{\Delta} \widetilde{R}_{\Delta} \widetilde{A}^{-1} \widetilde{R}_{\Delta}^{T} B_{\Delta}^{T}$ can be approximated by the following lumped block:

$$
M_{\lambda}^{-1}=B_{\Delta, D} \widetilde{R}_{\Delta} \widetilde{A} \widetilde{R}_{\Delta}^{T} B_{\Delta, D}^{T} .
$$

This leads to the lumped preconditioner

$$
M^{-1}=\left[\begin{array}{ll}
\frac{1}{h^{2}} I_{p_{\Gamma}} & \\
& M_{\lambda}^{-1}
\end{array}\right]
$$

for solving (4.1).

Remark 6.1. The mesh size $h$ is used in the above preconditioner. For applications where the mesh size is not explicitly provided and only the coefficient matrix in (2.3) 
is given, an estimate of $h$ can be obtained by comparing the nonzero entries in $A$ and $B$ blocks. From the definition of $A$ and $B$ for the incompressible Stokes problem (2.2), entries in $A$ and entries in $B$ have a difference of factor $h$ in general.

$M^{-1}$ is symmetric positive definite. Multiplication of $M^{-1}$ by a vector requires mainly the product of $\widetilde{A}$ with a vector. When the CG iteration is applied to solve the preconditioned system

$$
M^{-1} G\left[\begin{array}{c}
p_{\Gamma} \\
\lambda
\end{array}\right]=M^{-1} g
$$

with zero initial guess, all the iterates belong to the Krylov subspace generated by the operator $M^{-1} G$ and the vector $M^{-1} g$, which is also a subspace of the range of $M^{-1} G$. We denote the range of $M^{-1} G$ by $R_{M^{-1} G}$. The following lemma shows that the $\mathrm{CG}$ iteration applied to solving (6.1) cannot break down.

Lemma 6.2. Let the preconditioner $M^{-1}$ be symmetric positive definite. The $C G$ iteration applied to solving (6.1) with zero initial guess cannot break down.

Proof. We just need to show that for any $0 \neq x \in R_{M^{-1} G}, G x \neq 0$. Let $0 \neq x=$ $M^{-1} G y$ for a certain $y \in X$ and $y \neq 0 . G x=G M^{-1} G y$, which cannot be zero since $G y \neq 0$ and $y^{T} G M^{-1} G y \neq 0$.

Lemma 6.3. Let $M^{-1}$ be symmetric positive definite. For any $x=\left(p_{\Gamma}, \lambda\right) \in$ $R_{M^{-1} G}$,

$$
\langle M x, x\rangle=\max _{y \in R_{G}, y \neq 0} \frac{\langle y, x\rangle^{2}}{\left\langle M^{-1} y, y\right\rangle} .
$$

Proof. Denote the range of $M^{-\frac{1}{2}} G$ by $R_{M^{-1 / 2} G}$. For any $x \in R_{M^{-1} G}$,

$$
\begin{aligned}
\langle M x, x\rangle & =\left\langle M^{\frac{1}{2}} x, M^{\frac{1}{2}} x\right\rangle=\max _{z \in R_{M^{-1 / 2} G}, z \neq 0} \frac{\left\langle M^{\frac{1}{2}} x, z\right\rangle^{2}}{\langle z, z\rangle} \\
& =\max _{y \in R_{G}, y \neq 0} \frac{\left\langle M^{\frac{1}{2}} x, M^{-\frac{1}{2}} y\right\rangle^{2}}{\left\langle M^{-\frac{1}{2}} y, M^{-\frac{1}{2}} y\right\rangle}=\max _{y \in R_{G}, y \neq 0} \frac{\langle y, x\rangle^{2}}{\left\langle M^{-1} y, y\right\rangle} .
\end{aligned}
$$

In the following, we establish a condition number bound of the preconditioned operator $M^{-1} G$. We first have the following lemma.

Lemma 6.4. For any $v \in \widetilde{V}_{0}$,

$$
\left\langle M^{-1} B_{C} v, B_{C} v\right\rangle \leq C \Phi(H / h)\langle\widetilde{A} v, v\rangle,
$$

where $\Phi(H / h)$ is as defined in Lemma 5.5. We have

Proof. Given $v=\left(\mathbf{w}_{I}, q_{I}, \mathbf{w}_{\Delta}, \mathbf{w}_{\Pi}\right) \in \widetilde{V}_{0}$, let $g_{p_{\Gamma}}=B_{\Gamma I} \mathbf{w}_{I}+B_{\Gamma \Delta} \mathbf{w}_{\Delta}+B_{\Gamma \Pi} \mathbf{w}_{\Pi}$.

$$
\begin{aligned}
\left\langle M^{-1} B_{C} v, B_{C} v\right\rangle & =\frac{1}{h^{2}}\left\langle g_{p_{\Gamma}}, g_{p_{\Gamma}}\right\rangle+\left(B_{\Delta} \widetilde{R}_{\Delta} v\right)^{T} M_{\lambda}^{-1} B_{\Delta} \widetilde{R}_{\Delta} v \\
& =\frac{1}{h^{2}}\left\langle g_{p_{\Gamma}}, g_{p_{\Gamma}}\right\rangle+\left(B_{\Delta} \widetilde{R}_{\Delta} v\right)^{T} B_{\Delta, D} \widetilde{R}_{\Delta} \widetilde{A} \widetilde{R}_{\Delta}^{T} B_{\Delta, D}^{T}\left(B_{\Delta} \widetilde{R}_{\Delta} v\right) \\
& =\frac{1}{h^{2}}\left\langle g_{p_{\Gamma}}, g_{p_{\Gamma}}\right\rangle+\left\langle P_{D} v, P_{D} v\right\rangle_{\widetilde{A}} \\
& \leq \frac{1}{h^{2}}\left\langle g_{p_{\Gamma}}, g_{p_{\Gamma}}\right\rangle+C \Phi(H / h)\langle v, v\rangle_{\widetilde{A}},
\end{aligned}
$$

Copyright $@$ by SIAM. Unauthorized reproduction of this article is prohibited. 
where we used Lemma 5.5 for the last inequality. It is sufficient to bound the first term of the right-hand side in the above inequality.

We denote $\mathbf{w}=\left(\mathbf{w}_{I}, \mathbf{w}_{\Delta}, \mathbf{w}_{\Pi}\right) \in \widetilde{\mathbf{W}}$. Since $B_{I I} \mathbf{w}_{I}+B_{I \Delta} \mathbf{w}_{\Delta}+B_{I \Pi} \mathbf{w}_{\Pi}=0$ (cf. (5.2)), we have

$$
\begin{aligned}
\left\langle g_{p_{\Gamma}}, g_{p_{\Gamma}}\right\rangle & =\left[\begin{array}{l}
B_{I I} \mathbf{w}_{I}+B_{I \Delta} \mathbf{w}_{\Delta}+B_{I \Pi \mathbf{w}_{\Pi}} \\
B_{\Gamma I} \mathbf{w}_{I}+B_{\Gamma \Delta} \mathbf{w}_{\Delta}+B_{\Gamma \Pi} \mathbf{w}_{\Pi}
\end{array}\right]^{T}\left[\begin{array}{l}
B_{I I} \mathbf{w}_{I}+B_{I \Delta} \mathbf{w}_{\Delta}+B_{I \Pi \mathbf{w}_{\Pi}} \\
B_{\Gamma I} \mathbf{w}_{I}+B_{\Gamma \Delta} \mathbf{w}_{\Delta}+B_{\Gamma \Pi} \mathbf{w}_{\Pi}
\end{array}\right] \\
& =\langle\widetilde{B} \mathbf{w}, \widetilde{B} \mathbf{w}\rangle
\end{aligned}
$$

where $\widetilde{B}$ is defined in (5.4). From (2.6) and the stability of $\widetilde{B}$ (cf. Lemma 5.1), we have

$$
\begin{aligned}
\frac{1}{h^{2}}\left\langle g_{p_{\Gamma}}, g_{p_{\Gamma}}\right\rangle & =\frac{1}{h^{2}}\langle\widetilde{B} \mathbf{w}, \widetilde{B} \mathbf{w}\rangle \leq C\langle\widetilde{B} \mathbf{w}, \widetilde{B} \mathbf{w}\rangle_{Z^{-1}}=C \max _{q \in Q} \frac{\langle\widetilde{B} \mathbf{w}, q\rangle^{2}}{\langle q, q\rangle_{Z}} \\
& \leq C \max _{q \in Q} \frac{|\mathbf{w}|_{H^{1}}^{2}\|q\|_{L^{2}}^{2}}{\|q\|_{L^{2}}^{2}}=C|\mathbf{w}|_{H^{1}}^{2}=C\langle v, v\rangle_{\widetilde{A}},
\end{aligned}
$$

where for the last equality we used the fact that $B_{I I} \mathbf{w}_{I}+B_{I \Delta} \mathbf{w}_{\Delta}+B_{I \Pi} \mathbf{w}_{\Pi}=0$ and (5.3).

Lemma 6.5. For any given $y=\left(g_{p_{\Gamma}}, g_{\lambda}\right) \in R_{G}$, there exists $v \in \widetilde{V}_{0}$ such that $B_{C} v=y$ and $\langle\widetilde{A} v, v\rangle \leq \frac{C}{\beta^{2}}\left\langle M^{-1} y, y\right\rangle$.

Proof. Given $y=\left(g_{p_{\Gamma}}, g_{\lambda}\right) \in R_{G}$, take $\mathbf{w}_{\Delta}^{(I)}=B_{\Delta, D}^{T} g_{\lambda}$. Let $\mathbf{w}^{(I)}=\left(\mathbf{0}, \mathbf{w}_{\Delta}^{(I)}, \mathbf{0}\right) \in$ $\mathbf{W}_{I} \oplus \mathbf{W}_{\Delta} \oplus \mathbf{W}_{\Pi}$ and $v^{(I)}=\left(\mathbf{0}, 0, \mathbf{w}_{\Delta}^{(I)}, \mathbf{0}\right) \in \mathbf{W}_{I} \oplus Q_{I} \oplus \mathbf{W}_{\Delta} \oplus \mathbf{W}_{\Pi}$. We have

$$
\left|\mathbf{w}^{(I)}\right|_{H^{1}}^{2}=\left\langle A_{\Delta \Delta} \mathbf{w}_{\Delta}^{(I)}, \mathbf{w}_{\Delta}^{(I)}\right\rangle
$$

and

$$
B_{c} v^{(I)}=\left[\begin{array}{cccc}
B_{\Gamma I} & 0 & B_{\Gamma \Delta} & B_{\Gamma \Pi} \\
0 & 0 & B_{\Delta} & 0
\end{array}\right]\left[\begin{array}{c}
\mathbf{0} \\
0 \\
B_{\Delta, D}^{T} g_{\lambda} \\
\mathbf{0}
\end{array}\right]=\left[\begin{array}{c}
B_{\Gamma \Delta} \mathbf{w}_{\Delta}^{(I)} \\
g_{\lambda}
\end{array}\right],
$$

where we used the fact that $B_{\Delta} B_{\Delta, D}^{T}=I$.

We consider the solution to the following fully assembled system of linear equations of the form (2.3): find $\left(\mathbf{w}_{I}^{(I I)}, q_{I}^{(I I)}, \mathbf{w}_{\Gamma}^{(I I)}, q_{\Gamma}^{(I I)}\right) \in \mathbf{W}_{I} \bigoplus Q_{I} \bigoplus \mathbf{W}_{\Gamma} \bigoplus Q_{\Gamma}$ such that

$$
\left[\begin{array}{cccc}
A_{I I} & B_{I I}^{T} & A_{I \Gamma} & B_{\Gamma I}^{T} \\
B_{I I} & 0 & B_{I \Gamma} & 0 \\
A_{\Gamma I} & B_{I \Gamma}^{T} & A_{\Gamma \Gamma} & B_{\Gamma \Gamma}^{T} \\
B_{\Gamma I} & 0 & B_{\Gamma \Gamma} & 0
\end{array}\right]\left[\begin{array}{l}
\mathbf{w}_{I}^{(I I)} \\
q_{I}^{(I I)} \\
\mathbf{w}_{\Gamma}^{(I I)} \\
q_{\Gamma}^{(I I)}
\end{array}\right]=\left[\begin{array}{l}
\mathbf{0} \\
-B_{I \Delta} \mathbf{w}_{\Delta}^{(I)} \\
\mathbf{0} \\
g_{p_{\Gamma}}-B_{\Gamma \Delta} \mathbf{w}_{\Delta}^{(I)}
\end{array}\right],
$$

where a particular right-hand side is chosen. We first note that since $\left(g_{p_{\Gamma}}, g_{\lambda}\right) \in R_{G}$, the right-hand-side vector of the above system satisfies (cf. (4.5))

$\left(-B_{I \Delta} \mathbf{w}_{\Delta}^{(I)}\right)^{T} 1_{p_{I}}+\left(g_{p_{\Gamma}}-B_{\Gamma \Delta} \mathbf{w}_{\Delta}^{(I)}\right)^{T} 1_{p_{\Gamma}}=g_{p_{\Gamma}}^{T} 1_{p_{\Gamma}}-g_{\lambda}^{T} B_{\Delta, D}\left(B_{I \Delta}^{T} 1_{p_{I}}+B_{\Gamma \Delta}^{T} 1_{p_{\Gamma}}\right)=0$, i.e., it has zero average, which implies existence of the solution to (6.6). 
Denote $\mathbf{w}^{(I I)}=\left(\mathbf{w}_{I}^{(I I)}, \mathbf{w}_{\Gamma}^{(I I)}\right) \in \mathbf{W}$. From the inf-sup stability of the original problem (2.3) and Lemma 5.7, we have

$$
\left|\mathbf{w}^{(I I)}\right|_{H^{1}}^{2} \leq \frac{1}{\beta^{2}}\left\|\left[\begin{array}{l}
-B_{I \Delta} \mathbf{w}_{\Delta}^{(I)} \\
g_{p_{\Gamma}}-B_{\Gamma \Delta} \mathbf{w}_{\Delta}^{(I)}
\end{array}\right]\right\|_{Z^{-1}}^{2} \leq \frac{1}{\beta^{2}}\left\|\left[\begin{array}{l}
B_{I \Delta} \mathbf{w}_{\Delta}^{(I)} \\
B_{\Gamma \Delta} \mathbf{w}_{\Delta}^{(I)}
\end{array}\right]\right\|_{Z^{-1}}^{2}+\frac{1}{\beta^{2}}\left\|\left[\begin{array}{l}
0 \\
g_{p_{\Gamma}}
\end{array}\right]\right\|_{Z^{-1}}^{2} .
$$

The first term on the right-hand side of (6.7) can be bounded in the same way as done in (6.3), and we have

$$
\left\|\left[\begin{array}{c}
B_{I \Delta} \mathbf{w}_{\Delta}^{(I)} \\
B_{\Gamma \Delta} \mathbf{w}_{\Delta}^{(I)}
\end{array}\right]\right\|_{Z^{-1}}^{2} \leq C\left\langle A_{\Delta \Delta} \mathbf{w}_{\Delta}^{(I)}, \mathbf{w}_{\Delta}^{(I)}\right\rangle
$$

the second term can be bounded, using (2.6), by

$$
\left\|\left[\begin{array}{l}
0 \\
g_{p_{\Gamma}}
\end{array}\right]\right\|_{Z^{-1}}^{2} \leq \frac{C}{h^{2}}\left\langle g_{p_{\Gamma}}, g_{p_{\Gamma}}\right\rangle .
$$

Split the continuous subdomain boundary velocity $\mathbf{w}_{\Gamma}^{(I I)}$ into the dual part $\mathbf{w}_{\Delta}^{(I I)} \in$ $\mathbf{W}_{\Delta}$ and the primal part $\mathbf{w}_{\Pi}^{(I I)} \in \mathbf{W}_{\Pi}$, and denote $v^{(I I)}=\left(\mathbf{w}_{I}^{(I I)}, q_{I}^{(I I)}, \mathbf{w}_{\Delta}^{(I I)}, \mathbf{w}_{\Pi}^{(I I)}\right)$. We have from (6.6),

$$
\left[\begin{array}{llll}
B_{I I} & 0 & B_{I \Delta} & B_{I \Pi}
\end{array}\right]\left[\begin{array}{c}
\mathbf{w}_{I}^{(I I)} \\
q_{I}^{(I I)} \\
\mathbf{w}_{\Delta}^{(I I)} \\
\mathbf{w}_{\Pi}^{(I I)}
\end{array}\right]=-B_{I \Delta} \mathbf{w}_{\Delta}^{(I)}
$$

and

$$
B_{c} v^{(I I)}=\left[\begin{array}{cccc}
B_{\Gamma I} & 0 & B_{\Gamma \Delta} & B_{\Gamma \Pi} \\
0 & 0 & B_{\Delta} & 0
\end{array}\right]\left[\begin{array}{c}
\mathbf{w}_{I}^{(I I)} \\
q_{I}^{(I I)} \\
\mathbf{w}_{\Delta}^{(I I)} \\
\mathbf{w}_{\Pi}^{(I I)}
\end{array}\right]=\left[\begin{array}{c}
g_{p_{\Gamma}}-B_{\Gamma \Delta} \mathbf{w}_{\Delta}^{(I)} \\
0
\end{array}\right]
$$

Let $v=v^{(I)}+v^{(I I)}$. We can see from (6.10) that $v \in \widetilde{V}_{0}$; cf. (5.2). We can also see from (6.5) and (6.11) that $B_{C} v=y$. Furthermore, by (5.3),

$|v|_{\widetilde{A}}^{2}=\left|\mathbf{w}^{(I)}+\mathbf{w}^{(I I)}\right|_{H^{1}}^{2} \leq\left|\mathbf{w}^{(I)}\right|_{H^{1}}^{2}+\left|\mathbf{w}^{(I I)}\right|_{H^{1}}^{2} \leq \frac{C}{\beta^{2}}\left\langle A_{\Delta \Delta} \mathbf{w}_{\Delta}^{(I)}, \mathbf{w}_{\Delta}^{(I)}\right\rangle+\frac{C}{\beta^{2} h^{2}}\left\langle g_{p_{\Gamma}}, g_{p_{\Gamma}}\right\rangle$,

where we used (6.4), (6.7), (6.8), and (6.9) for the last inequality.

On the other hand, we have

$$
\begin{aligned}
\left\langle M^{-1} y, y\right\rangle & =\frac{1}{h^{2}}\left\langle g_{p_{\Gamma}}, g_{p_{\Gamma}}\right\rangle+g_{\lambda}^{T} M_{1, \lambda}^{-1} g_{\lambda}=\frac{1}{h^{2}}\left\langle g_{p_{\Gamma}}, g_{p_{\Gamma}}\right\rangle+g_{\lambda}^{T} B_{\Delta, D} \widetilde{R}_{\Delta} \widetilde{A} \widetilde{R}_{\Delta}^{T} B_{\Delta, D}^{T} g_{\lambda} \\
& =\frac{1}{h^{2}}\left\langle g_{p_{\Gamma}}, g_{p_{\Gamma}}\right\rangle+\left\langle A_{\Delta \Delta} \mathbf{w}_{\Delta}^{(I)}, \mathbf{w}_{\Delta}^{(I)}\right\rangle \cdot
\end{aligned}
$$

We also need the following lemma. 
Lemma 6.6. For any $v=\left(\mathbf{w}_{I}, p_{I}, \mathbf{w}_{\Delta}, \mathbf{w}_{\Pi}\right) \in \widetilde{V}_{0}, B_{C} v \in R_{G}$.

Proof. We know that for any $\left(\mathbf{f}_{I}, \mathbf{f}_{\Delta}, \mathbf{f}_{\Pi}\right) \in \mathbf{W}_{I} \oplus \mathbf{W}_{\Delta} \oplus \mathbf{W}_{\Pi}, g$ defined by (4.3) is in $R_{G}$. For any $v=\left(\mathbf{w}_{I}, p_{I}, \mathbf{w}_{\Delta}, \mathbf{w}_{\Pi}\right) \in \widetilde{V}_{0}$, from the definition of $\widetilde{A}$ in (4.4), there always exists $\left(\mathbf{f}_{I}, \mathbf{f}_{\Delta}, \mathbf{f}_{\Pi}\right) \in \mathbf{W}_{I} \oplus \mathbf{W}_{\Delta} \oplus \mathbf{W}_{\Pi}$ such that

$$
\widetilde{A} v=\left[\begin{array}{c}
\mathbf{f}_{I} \\
0 \\
\mathbf{f}_{\Delta} \\
\mathbf{f}_{\Pi}
\end{array}\right], \quad \text { i.e., } \quad v=\widetilde{A}^{-1}\left[\begin{array}{c}
\mathbf{f}_{I} \\
0 \\
\mathbf{f}_{\Delta} \\
\mathbf{f}_{\Pi}
\end{array}\right] .
$$

Taking such $\left(\mathbf{f}_{I}, \mathbf{f}_{\Delta}, \mathbf{f}_{\Pi}\right), g$ defined in $(4.3)$ is $B_{C} v$.

The following lemma is an immediate result of Lemmas 6.5 and 6.6.

Lemma 6.7. The space $R_{G}$ is the same as the range of $B_{C}$ applied on $\widetilde{V}_{0}$.

The condition number bound of the preconditioned operator $M^{-1} G$ is given in the following theorem.

Theorem 6.8. For all $x=\left(p_{\Gamma}, \lambda\right) \in R_{M^{-1} G}$,

$$
c \beta^{2}\langle M x, x\rangle \leq\langle G x, x\rangle \leq C \Phi(H / h)\langle M x, x\rangle .
$$

Here, for two-dimensional problems, $\Phi(H / h)=(H / h)(1+\log (H / h))$ when only subdomain corner velocities are chosen as coarse level primal variables; $\Phi(H / h)=H / h$ when both subdomain corner and edge-average velocity degrees of freedom are chosen as primal variables.

Proof.

$$
\langle G x, x\rangle=x^{T} B_{C} \widetilde{A}^{-1} B_{C}^{T} x=x^{T} B_{C} \widetilde{A}^{-1} \widetilde{A} \widetilde{A}^{-1} B_{C}^{T} x=\left\langle\widetilde{A}^{-1} B_{C}^{T} x, \widetilde{A}^{-1} B_{C}^{T} x\right\rangle_{\widetilde{A}} .
$$

Since $\widetilde{A}^{-1} B_{C}^{T} x \in \widetilde{V}_{0}$ and $\langle\cdot, \cdot\rangle_{\widetilde{A}}$ defines an inner product on $\widetilde{V}_{0}$, we have

$$
\langle G x, x\rangle=\max _{v \in \widetilde{V}_{0}, v \neq 0} \frac{\left\langle v, \widetilde{A}^{-1} B_{C}^{T} x\right\rangle_{\widetilde{A}}^{2}}{\langle v, v\rangle_{\widetilde{A}}}=\max _{v \in \widetilde{V}_{0}, v \neq 0} \frac{\left\langle B_{C} v, x\right\rangle^{2}}{\langle\widetilde{A} v, v\rangle} .
$$

Lower bound. From Lemma 6.5, we know that for any given $y=\left(g_{p_{\Gamma}}, g_{\lambda}\right) \in R_{G}$, there exits $v \in \widetilde{V}_{0}$ such that $B_{C} v=y$ and $\langle\widetilde{A} v, v\rangle \leq \frac{C}{\beta^{2}}\left\langle M^{-1} y, y\right\rangle$. Then from (6.12), we have

$$
\langle G x, x\rangle \geq \frac{\left\langle B_{C} v, x\right\rangle^{2}}{\langle\widetilde{A} v, v\rangle} \geq c \beta^{2} \frac{\langle y, x\rangle^{2}}{\left\langle M^{-1} y, y\right\rangle} .
$$

Since $y$ is arbitrary, using Lemma 6.3 , we have

$$
\langle G x, x\rangle \geq c \beta^{2} \max _{y \in R_{G}, y \neq 0} \frac{\langle y, x\rangle^{2}}{\left\langle M^{-1} y, y\right\rangle}=c \beta^{2}\langle M x, x\rangle .
$$

Upper bound. From (6.12), Lemmas 6.4, 6.7, and 6.3, we have

$$
\begin{aligned}
\langle G x, x\rangle & \leq C \Phi(H / h) \max _{v \in \widetilde{V}_{0}, v \neq 0} \frac{\left\langle B_{C} v, x\right\rangle^{2}}{\left\langle M^{-1} B_{C} v, B_{C} v\right\rangle} \\
& =C \Phi(H / h) \max _{y \in R_{G}, y \neq 0} \frac{\langle y, x\rangle^{2}}{\left\langle M^{-1} y, y\right\rangle}=C \Phi(H / h)\langle M x, x\rangle .
\end{aligned}
$$

Copyright $\odot$ by SIAM. Unauthorized reproduction of this article is prohibited. 
Remark 6.9. The upper bound $\Phi(H / h)$ can be improved when the Dirichlet preconditioner is used in the algorithm. However, using the Dirichlet preconditioner requires more computational cost in each iteration and more complicated coarse level space in the algorithm for solving incompressible Stokes problems; cf. [22]. For example, for two-dimensional problems, besides the subdomain corner velocities, certain subdomain edge-average velocities are also required in the coarse level space and the upper bound $\Phi(H / h)=(1+\log (H / h))^{2}$ can be obtained in Theorem 6.8. Application of the Dirichlet preconditioner in the proposed algorithm is addressed in [34] by the authors.

Remark 6.10. For three-dimensional problems, the same bound as in Theorem 6.8 can be proved, where $\Phi(H / h)=(H / h)(1+\log (H / h))$, when the coarse space is spanned by both subdomain corner and edge-average velocities; cf. Remark 5.6. Since only the lumped preconditioner is considered in this paper, the additional coarse level components, as discussed in [22], corresponding to the divergence-free constraints across subdomain boundaries and required for using the Dirichlet preconditioner, are no longer needed here.

Remark 6.11. With only minor modifications, the algorithm proposed in this paper and its analysis apply equally well to the discontinuous pressure case. In that situation, $p_{\Gamma}$ and the blocks related to it in (3.1) can simply be replaced by the vector containing subdomain constant pressures and its corresponding blocks, respectively. The formulation of the algorithm then follows as presented in section 4, and the same bound as in Theorem 6.8 will be obtained. Note in particular that the bound obtained in Theorem 6.8 is for the lumped preconditioner case, which is different from the quasi-optimal bound obtained previously by other authors, e.g., [22], corresponding to using the Dirichlet preconditioner. Numerical experiments of our algorithm for the discontinuous pressure case will also be reported in the next section.

Remark 6.12. The same condition number bound has been proved by Kim and Lee [14] and Kim, Lee, and Park [12] for their FETI-DP algorithms with lumped preconditioner for solving incompressible Stokes equations. In their algorithms, discontinuous pressure is considered and their approaches do not apply to the continuous pressure case.

7. Numerical experiments. We consider solving the incompressible Stokes problem (2.1) in the square domain $\Omega=[0,1] \times[0,1]$. Zero Dirichlet boundary conditions are used. The right-hand-side function $\mathbf{f}$ is chosen such that the exact solution is

$$
\mathbf{u}=\left[\begin{array}{c}
\sin ^{3}(\pi x) \sin ^{2}(\pi y) \cos (\pi y) \\
-\sin ^{2}(\pi x) \sin ^{3}(\pi y) \cos (\pi x)
\end{array}\right] \text { and } p=x^{2}-y^{2} .
$$

The modified Taylor-Hood mixed finite element, as shown in Figure 2.1, is used for the finite element solution. The preconditioned system (6.1) is solved by the CG iteration; the iteration is stopped when the $L^{2}$-norm of the residual is reduced by a factor of $10^{-6}$.

Table 7.1 shows the minimum and maximum eigenvalues of the iteration matrix $M^{-1} G$ and the iteration counts. Two different coarse level spaces are tested in the experiments: the coarse space spanned by only the subdomain corner velocities and the coarse space spanned by both the subdomain corner and the subdomain edgeaverage velocities. Even though the edge-average velocities are not required in the coarse space for the analysis, including them in the coarse level problem improves 
TABLE 7.1

Performance of solving (6.1) with different coarse spaces using continuous pressures.

\begin{tabular}{|c|c|c|c|c|c|c|c|}
\hline \multirow[b]{2}{*}{$H / h$} & \multirow[b]{2}{*}{ \#sub } & \multicolumn{3}{|c|}{ Corner velocities only } & \multicolumn{3}{|c|}{ Corner and edge-averages } \\
\hline & & $\lambda_{\min }$ & $\lambda_{\max }$ & Iter & $\lambda_{\min }$ & $\lambda_{\max }$ & Iter \\
\hline \multirow[t]{5}{*}{8} & $4 \times 4$ & 0.35 & 8.92 & 21 & 0.36 & 2.82 & 16 \\
\hline & $8 \times 8$ & 0.35 & 10.07 & 28 & 0.36 & 2.83 & 16 \\
\hline & $16 \times 16$ & 0.35 & 10.23 & 29 & 0.36 & 2.83 & 17 \\
\hline & $24 \times 24$ & 0.35 & 10.30 & 29 & 0.36 & 2.83 & 17 \\
\hline & $32 \times 32$ & 0.35 & 10.33 & 29 & 0.36 & 2.83 & 17 \\
\hline \#sub & $H / h$ & $\lambda_{\min }$ & $\lambda_{\max }$ & Iter & $\lambda_{\min }$ & $\lambda_{\max }$ & Iter \\
\hline \multirow[t]{5}{*}{$8 \times 8$} & 4 & 0.30 & 4.22 & 21 & 0.33 & 2.91 & 18 \\
\hline & 8 & 0.35 & 10.07 & 28 & 0.36 & 2.83 & 16 \\
\hline & 16 & 0.35 & 24.22 & 36 & 0.36 & 3.54 & 17 \\
\hline & 24 & 0.35 & 40.12 & 43 & 0.36 & 5.27 & 19 \\
\hline & 32 & 0.35 & 57.15 & 50 & 0.36 & 7.05 & 22 \\
\hline
\end{tabular}
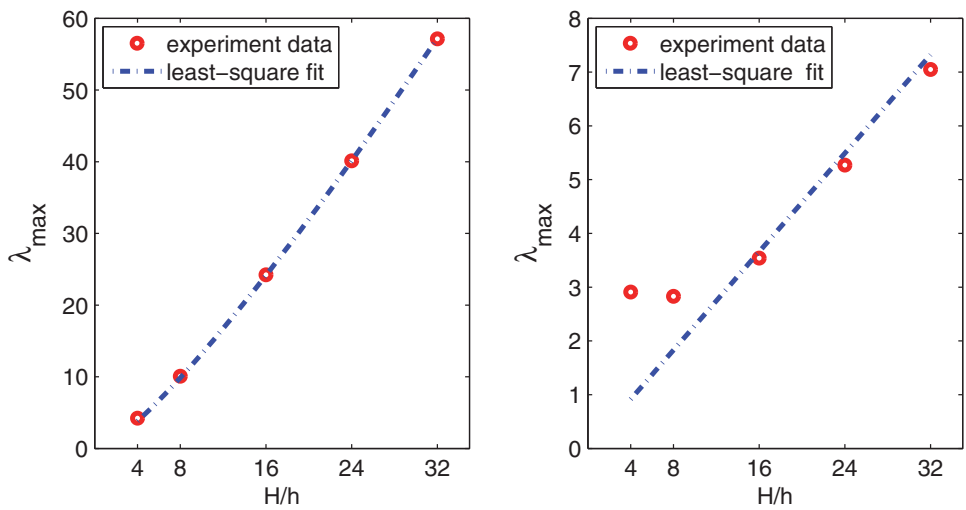

FIG. 7.1. Least-square fit of $\lambda_{\max }$ in Table 7.1 by $(H / h)(1+\log (H / h))$ (left) when the coarse space spanned by only the subdomain corner velocities and by $H / h$ (right) when the coarse space spanned by both the subdomain corner and the subdomain edge-average velocities.

the convergence rate. We can see from Table 7.1 that the minimum eigenvalue is independent of the mesh size in both cases. The maximum eigenvalue is independent of the number of subdomains for fixed $H / h$; for a fixed number of subdomains, it depends on $H / h$ in the order of $(H / h)(1+\log (H / h))$ for the case when the coarse space is spanned by only the subdomain corner velocities and in the order of $H / h$ when the coarse space spanned by both the subdomain corner and the subdomain edge-average velocities; cf. Theorem 6.8. Plots of the least-square fit of the maximum eigenvalues by $(H / h)(1+\log (H / h))$ and $H / h$ for both cases, respectively, are shown in Figure 7.1.

Table 7.2 shows the performance of our algorithm for solving the same problem but by using a mixed finite element with discontinuous pressure. We use a uniform mesh of triangles, shown on the left in Figure 7.2; the velocity finite element space contains the piecewise linear functions on the mesh and the pressure is a constant on each union of four triangles as shown on the right in the figure. The same mixed finite element has also been used in [22]. 
TABle 7.2

Performance of solving (6.1) with different coarse spaces using discontinuous pressures.

\begin{tabular}{|c|c|c|c|c|c|c|c|}
\hline \multirow[b]{2}{*}{$H / h$} & \multirow[b]{2}{*}{ \#sub } & \multicolumn{3}{|c|}{ Corner velocities only } & \multicolumn{3}{|c|}{ Corner and edge-averages } \\
\hline & & $\lambda_{\min }$ & $\overline{\lambda_{\max }}$ & Iter & $\overline{\lambda_{\min }}$ & $\overline{\lambda_{\max }}$ & Iter \\
\hline \multirow[t]{5}{*}{8} & $4 \times 4$ & 0.48 & 7.93 & 22 & 0.50 & 2.31 & 13 \\
\hline & $8 \times 8$ & 0.48 & 9.00 & 25 & 0.50 & 2.31 & 13 \\
\hline & $16 \times 16$ & 0.48 & 9.20 & 25 & 0.50 & 2.32 & 14 \\
\hline & $24 \times 24$ & 0.48 & 9.20 & 25 & 0.50 & 2.32 & 14 \\
\hline & $32 \times 32$ & 0.48 & 9.21 & 25 & 0.50 & 2.31 & 14 \\
\hline \#sub & $H / h$ & $\lambda_{\min }$ & $\lambda_{\max }$ & Iter & $\lambda_{\min }$ & $\lambda_{\max }$ & Iter \\
\hline \multirow[t]{5}{*}{$8 \times 8$} & 4 & 0.41 & 3.91 & 19 & 0.47 & 2.05 & 13 \\
\hline & 8 & 0.48 & 9.00 & 25 & 0.50 & 2.31 & 13 \\
\hline & 16 & 0.49 & 21.39 & 36 & 0.49 & 3.30 & 16 \\
\hline & 24 & 0.50 & 35.56 & 43 & 0.49 & 4.89 & 19 \\
\hline & 32 & 0.50 & 50.87 & 50 & 0.48 & 6.58 & 21 \\
\hline
\end{tabular}
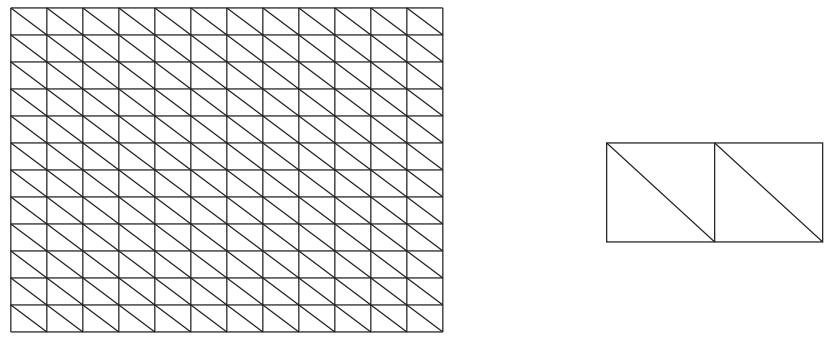

FIG. 7.2. The mesh and the mixed finite element.

Comparing Table 7.1 with Table 7.2, we can see that the convergence rates of our algorithm, using either continuous or discontinuous pressure, are quite similar.

Acknowledgments. The authors are very grateful to Olof Widlund and Clark Dohrmann for their suggestion of this problem.

\section{REFERENCES}

[1] H. Benhassine and A. Bendali, A non-overlapping domain decomposition method for continuous-pressure mixed finite element approximations of the Stokes problem, Math. Model. Numer. Anal., 45 (2011), pp. 675-696.

[2] D. Braess, Finite Elements, 2nd ed., Cambridge University Press, Cambridge, UK, 2001.

[3] C. R. Dohrmann, Preconditioning of saddle point systems by substructuring and a penalty approach, in Lect. Notes Comput. Sci. Eng. 55, Springer, New York, 2006, pp. 53-64.

[4] C. R. Dohrmann, An approximate BDDC preconditioner, Numer. Linear Algebra Appl., 14 (2007), pp. 149-168.

[5] C. R. Dohrmann and O. B. Widlund, An overlapping Schwarz algorithm for almost incompressible elasticity, SIAM J. Numer. Anal., 47 (2009), pp. 2897-2923.

[6] C. R. Dohrmann and O. B. Widlund, Hybrid domain decomposition algorithms for compressible and almost incompressible elasticity, Internat. J. Numer. Methods Engrg., 82 (2010), pp. $157-183$.

[7] C. Farhat And F. X. Roux, A method of finite element tearing and interconnecting and its parallel solution algorithm, Internat. J. Numer. Meths. Engrg., 32 (1991), pp. 1205-1227.

Copyright (C) by SIAM. Unauthorized reproduction of this article is prohibited. 
[8] C. Farhat, J. Mandel, and F. X. Roux, Optimal convergence properties of the FETI domain decomposition method, Comput. Methods Appl. Mech. Engrg., 115 (1994), pp. 367-388.

[9] P. Goldfeld, Balancing Neumann-Neumann Preconditioners for the Mixed Formulation of Almost-Incompressible Linear Elasticity, Technical report TR2003-847, Courant Institute of Mathematical Sciences, New York University, 2003.

[10] P. Goldfeld, L. Pavarino, And O. B. Widlund, Balancing Neumann-Neumann preconditioners for mixed approximations of heterogeneous problems in linear elasticity, Numer. Math., 95 (2003), pp. 283-324.

[11] H. H. KIM AND C.-O. LEE, A Neumann-Dirichlet preconditioner for a FETI-DP formulation of the two-dimensional Stokes problem with mortar methods, SIAM J. Sci. Comput., 28 (2006), pp. 1133-1152.

[12] H. H. KIM, C.-O. LEE, AND E.-H. PARK, A FETI-DP formulation for the Stokes problem without primal pressure components, SIAM J. Numer. Anal., 47 (2010), pp. 4142-4162.

[13] H. H. Kim And C.-O. LeE, A two-level nonoverlapping Schwarz algorithm for the Stokes problem without primal pressure unknowns, Internat. J. Numer. Methods Engrg., 88 (2011), pp. $1390-1410$.

[14] H. H. KIm AND C.-O. LeE, A FETI-DP formulation for the three-dimensional Stokes problem without primal pressure unknowns, SIAM J. Sci. Comput., 28 (2010), pp. 3301-3322.

[15] H. H. KIM AND X. TU, A three-level BDDC algorithm for mortar discretization, SIAM J. Numer. Anal., 47 (2009), pp. 1576-1600.

[16] A. Klawonn and L. F. PaVarino, Overlapping Schwarz methods for mixed linear elasticity and Stokes problems, Comput. Methods Appl. Mech. Engrg., 165 (1998), pp. 233-245.

[17] A. Klawonn and O. Rheinbach, Inexact FETI-DP methods, Internat. J. Numer. Methods Engrg., 69 (2007), pp. 284-307.

[18] A. Klawonn and O. B. Widlund, Dual-primal FETI methods for linear elasticity, Comm. Pure Appl. Math., 59 (2006), pp. 1523-1572.

[19] A. Klawonn, O. B. Widlund, and M. DrYja, Dual-primal FETI methods for threedimensional elliptic problems with heterogeneous coefficients, SIAM J. Numer. Anal., 40 (2002), pp. 159-179.

[20] J. LI, A dual-primal FETI method for incompressible Stokes equations, Numer. Math., 102 (2005), pp. 257-275.

[21] J. Li And O. B. WidLund, FETI-DP, BDDC, and block Cholesky methods, Internat. J. Numer. Methods Engrg., 66 (2006), pp. 250-271.

[22] J. Li AND O. B. WidLund, BDDC algorithms for incompressible Stokes equations, SIAM J. Numer. Anal., 44 (2006), pp. 2432-2455.

[23] J. Li AND O. B. WidLund, On the use of inexact subdomain solvers for BDDC algorithms, Comput. Methods Appl. Mech. Engrg., 196 (2007), pp. 1415-1428.

[24] L. F. PaVArino And O. B. Widlund, Balancing Neumann-Neumann methods for incompressible Stokes equations, Comm. Pure Appl. Math., 55 (2002), pp. 302-335.

[25] L. F. Pavarino, O. B. Widlund, And S. Zampini, BDDC preconditioners for spectral element discretizations of almost incompressible elasticity in three dimensions, SIAM J. Sci. Comput., 32 (2010), pp. 3604-3626.

[26] J. Š́́stek, B. Sousedik, P. Burda, J. Mandel, And J. Novotny, Application of the parallel BDDC preconditioner to the Stokes flow, Comput. \& Fluids, 46 (2011), pp. 429-435.

[27] C. TAYlor And P. Hood, Numerical solution of the Navier-Stokes equations using the finite element technique, Comput. \& Fluids, 1 (1973), pp. 1-28.

[28] X. TU, A BDDC algorithm for a mixed formulation of flows in porous media, Electron. Trans. Numer. Anal., 20 (2005), pp. 164-179.

[29] X. TU, A BDDC algorithm for flow in porous media with a hybrid finite element discretization, Electron. Trans. Numer. Anal., 26 (2007), pp. 146-160.

[30] X. Tu, Three-level BDDC, in Lect. Notes Comput. Sci. Eng. 55, Springer, New York, 2006, pp. 437-444.

[31] X. Tu, Three-level BDDC in two dimensions, Internat. J. Numer. Methods Engrg., 69 (2007), pp. $33-59$.

[32] X. TU, Three-level BDDC in three dimensions, SIAM J. Sci. Comput., 29 (2007), pp. 1759-1780.

[33] X. TU, A three-level BDDC algorithm for saddle point problems, Numer. Math., 119 (2011), pp. $189-217$.

[34] X. TU AND J. LI, A unified dual-primal finite element tearing and interconnecting approach for incompressible Stokes equations, Internat. J. Numer. Methods Engrg., 94 (2013), pp. $128-149$.

[35] A. Toselli and O. B. Widlund, Domain Decomposition Methods: Algorithms and Theory, Springer, Berlin, 2004.

Copyright (c) by SIAM. Unauthorized reproduction of this article is prohibited. 